\title{
Full Insurance, Asymmetric Information and Genetic Testing
}

\author{
Jose S. Penalva Zuasti* \\ Department of Economic and Business, Universitat Pompeu Fabra \\ Ramon Trias Fargas, 25-27, Barcelona, 08005, SPAIN \\ E-mail: jose.penalva@econ.upf.es
}

Key Words: insurance, asymmetric information, genetic testing, portfolio choice.

\begin{abstract}
This paper extends previous results on optimal insurance trading in the presence of a stock market that allows continuous asset trading and substantial personal heterogeneity, and applies those results in a context of asymmetric information with references to the role of genetic testing in insurance markets.

We find a novel and surprising result under symmetric information: agents may optimally prefer to purchase full insurance despite the presence of unfairly priced insurance contracts, and other assets which are correlated with insurance.

Asymmetric information has a Hirschleifer-type effect which can be solved by suspending insurance trading. Nevertheless, agents can attain their first best allocations, which suggests that the practice of restricting insurance not to be contingent on genetic tests can be efficient.
\end{abstract}

\section{INSURANCE AND THE MARKET}

In some states in the U.S., as in most countries, insurance companies are explicitly forbidden from using genetic tests in determining the coverage and prices offered to consumers. In contrast, the U.K. government has taken a pioneering role in allowing life insurers to use a limited num-

\footnotetext{
* I would like to thank Bryan Ellickson for the idea of using marked point processes for modelling genetic testing as well as his support and suggestions. I would also like to thank Alberto Bisin and William Zame for their questions, comments and suggestions that have helped shape this paper. This paper has been presented at the Universitat Pompeu Fabra, the Insurance: Mathematics and Economics conference, the VIII Foro de Finanzas, and the University of Rochester. I thank the audiences for helpful comments and discussion. I also wish to acknowledge financial support from the Principal International España S.A., the Spanish Ministerio de Educación y Cultura (DEGSIC, PB98-1057), and the Universitat Pompeu Fabra (COFREA99.003). All errors remain my own.
} 
ber of genetic tests in setting rates. It is too early to tell what the result will be but there is and has been for some time a debate as to whether such genetic 'screening' should be allowed.

This paper considers one aspect of this debate, namely the importance of incorporating the information gathered from genetic testing into insurance transactions. We find it is important to extend previous insurance models to incorporate the effect of having actively traded exchanges, such as stock markets and insurance derivatives markets (Chicago, Bermuda). These exchanges allow continuous trading and full diversification. We also incorporate substantial personal heterogeneity in our model. First, we analyze the extended insurance model under full information and complete markets, and we find substantial differences in agents' insurance behaviour with respect to static models. Then, we introduce asymmetric information and consider a way to design insurance markets in an efficient manner and in a way that ensures equilibrium exists. We find that if one only takes account of purely informational reasons, the steps taken by the U.K. government are not justified.

Allowing for continuously traded securities is not a trivial extension of classical insurance models and leads to different conclusions. Classic insurance theory states: agents will never find it optimal to purchase full insurance in the presence of positive loadings - i.e. if the price of insurance was higher than its expected value (also called its fair price). We find that permitting dynamic trading in some assets alters this classical result. The extra trading opportunities imply that a smaller number of assets will be required in order to complete the market (Duffie and Huang (1985), Penalva(1999)). According to some results in the literature (Smith and Mayers(1983) for example), the introduction of additional securities could affect agents' demand for insurance in unpredictable ways. We find that insurance demands in modern economies with active and continuously open exchanges are quite predictable. In fact, optimal insurance demand is: buy and hold full insurance. This insurance demand is independent of the returns of other assets in the economy and whether insurance in fairly priced or not. The effect of unfair pricing of insurance is incorporated into the agent's problem as a reduction in wealth which then affects optimal consumption. This optimal consumption will not depend on agent-specific events (accidents) but on aggregate scarcity, which drives asset prices. Optimal consumption will be attained by adjusting wealth using actively traded fully diversified assets.

Once the properties of modern insurance markets have been established we introduce asymmetric information. We only consider purely agentspecific information. From the results of Grossman and Stiglitz(1980) we know that markets will not be informationally efficient and hence will 
not be willing to pay the costs of genetic testing. The cost of the tests is a net social welfare loss. In our analysis we show that this is because this information has no value per se, as in Milgrom and Stokey(1982). The new information will just increase the price of insurance for some agents and reduce it for others without any efficiency gains. We consider the effect of restricting insurance trading not to incorporate genetic information and find that it could be a potentially efficient solution to the problems caused by asymmetric information.

\section{THE MODEL}

In our economy there are two consumption dates, $t \in\{0,1\}$, and $n<$ $\infty$ agents indexed by $i \in I$, all of which have preferences described by von Neumann-Morgenstern expected utility:

$$
U_{i}(x)=u_{i}(x(0))+\beta_{i} E\left(u_{i}(x(1))\right) .
$$

Agents' endowments, $e_{i}$, are of the classical insurance type: $w_{i}$ if no accident (event $\neg A_{i}$ ), and $w_{i}-L$ in case of an accident (event $A_{i}$ ). We assume that agents have increasing, differentiable and strictly concave Bernoulli utility functions. Also, let $e$ denote the aggregate endowment and assume that $e>0$ (or equivalently that $\sum_{i} w_{i}-n L>0$ ).

The initial probability that agent $i$ suffers an accident is $p_{i}$. Assume there is an interval of length one of time in which accidents can occur - prior to the consumption date. Then, if an agent suffers an accident it will be immediately and publicly announced. We want to allow for the possibility that the probability of an accident depends on the total number of accidents. This generalization may be important if one wants to incorporate the effects of contagion of infectious diseases (more accidents, more risk), or technological advances in treatment which depend on the extension of the disease (more accidents, less risk).

Let $N^{e}(t)$ denote the number of agents who have suffered an accident up to date $t \in[0,1], N^{e}(t)=\sum_{i \in I} N_{i}(t)$. Assume also that the arrival time of the accident is given by an exponential distribution with parameter $\lambda_{i}$ which depends on $N^{e}(t), \lambda_{i}\left(N^{e}(t)\right)$; accident occurrences are independent events, conditional on the aggregate number of accidents up to that time, $N^{e}(t)$. Let $k$ be the number of different types of agents according to their hazard function, $\lambda_{i}\left(N^{e}(t)\right)$. For $j=1, \ldots, k$, let $I_{j}$ be the index of agents that have the same hazard function, $\lambda_{j}\left(N^{e}(t)\right)$. As we shall see below, this parameter, $k$, is the only heterogeneity parameter that plays an important role in the analysis. Naturally, in the special case where there are no population effects: $\lambda_{i}\left(N^{e}(t)\right)=\lambda_{i} \in \mathbf{R}$, then

$$
p_{i}=1-\exp ^{-\lambda_{i}} \Leftrightarrow \quad \lambda_{i}=-\ln \left(1-p_{i}\right) .
$$


All information is common knowledge and costless. All agents act as price takers.

For purposes of illustration we will consider an economy with 10 agents, five of type A and five of type B, with quadratic preferences parametrized as:

$$
\begin{gathered}
U_{i}(x)=E\left(u_{i}(x)\right) ; \quad u_{i}(x)=\frac{1}{2}\left(x-\bar{c}_{i}\right)^{2} . \\
\frac{\left|\bar{c}_{i}\right| w_{i}|L| \lambda_{i}\left|p_{i}\right|}{\frac{\mid \text { Type A }|20| 10|5| 0.02532|0.025|}{\mid \text { Type B }|30| 15|5| 0.07796|0.075|}}
\end{gathered}
$$

We impose that agent's accidents are independent for ease of computation although all the results in the paper apply to the more general case (where the hazard can depend on the aggregate number of realized accidents).

\section{INSURANCE DEMAND}

\subsection{The Static Economy}

Classical insurance theory (Malinvaud(1972), Borch(1990), Cass, Chichilninski, and $\mathrm{Wu}(1996, \mathrm{CCW}), \ldots)$ considers the case in which trade occurs before accidents can occur and there are no opportunities to retrade until spot markets open and all information has been revealed, i.e. at date one. We refer to this as the static economy. The standard insurance demand problem is to decide how much coverage, $\alpha$, to buy if for each $S_{i}$ units of consumption you spend in insurance you receive $L$ units of consumption if you suffer an accident. Standard insurance demand says that an agent of type A will purchase full coverage if and only if the price of insurance is 0.125 , its fair price. If the price were below its fair price, say 0.120 , she will overinsure (buy 1.08 units of insurance) while if the price is unfair, say 0.130, she will underinsure (buy 0.92 units).

But even in the simplest case, with $\lambda_{i}\left(N^{e}(t)\right)=\lambda_{i}$, the economy has much more uncertainty than just $A_{i}$ and $\neg A_{i}$. Uncertainty is given by the $2^{10}=1,024$ possible realizations of accidents or no accidents to each of the 10 agents. Denote the states of the world by $\omega$ and let $\Omega$ be the set of all possible $\omega$. The $\lambda_{i}(\cdot)$ 's induce a probability on $\left(\Omega, 2^{\Omega}\right)$ denoted by $P$. The consumption space is $L_{+}(\Omega)$ the set of all positive $2^{\Omega}$-measurable functions. Such economies have well known properties, in particular our parameterized economy has the equilibrium in statecontingent commodity trading $\left(\pi,\left(x_{i}\right)_{i}\right)$ given in Table 1. 


\begin{tabular}{|c|ccccccccccc|}
\hline$N^{e}(1, \omega)$ & 0 & 1 & 2 & 3 & 4 & 5 & 6 & 7 & 8 & 9 & 10 \\
\hline$\pi(\omega)$ & 1.00 & 1.04 & 1.08 & 1.12 & 1.16 & 1.20 & 1.24 & 1.28 & 1.32 & 1.36 & 1.40 \\
\hline$\xi(\omega)$ & 0.98 & 1.02 & 1.06 & 1.10 & 1.14 & 1.18 & 1.22 & 1.25 & 1.29 & 1.33 & $1.37 \mid$ \\
\hline$x_{A}(\omega)$ & 10.08 & 9.68 & 9.28 & 8.89 & 8.49 & 8.09 & 7.69 & 7.30 & 6.90 & 6.50 & 6.11 \\
\hline$x_{B}(\omega)$ & 14.92 & 14.32 & 13.72 & 13.11 & 12.51 & 11.91 & 11.31 & 10.70 & 10.10 & 9.50 & 8.89 \\
\hline
\end{tabular}

TABLE 1.

State-contingent Walrasian Equilibrium

As is illustrated on Table 1, equilibrium prices ( $\pi$, renormalized to $\xi$ ) and optimal demands, $x_{i}$, are a function of $N^{e}$, the total number of accidents in the economy. This corresponds to the well-known property of optimal risk-sharing or the existence of a representative agent (Wilson(1968), Huang(1987)).

Several things to note about this economy is the equilibrium price of insurance is not fair: 0.130 for type A and 0.389 for type B, which represent insurance loadings ${ }^{1}$ of $3.8 \%$ and $3.6 \%$ respectively. This would suggest that agents would be underinsuring. On the other hand, insurance contracts (in the static economy) are insufficient to span the $2^{10}$ states of the world so that other assets need to be introduced. CCW showed that there is sufficient symmetry to reduce the number of required assets. In particular, each agent would require $2 \times 11=22 \mathrm{mu}-$ tual insurance contracts ${ }^{2}$ and 11 Arrow securities (one for each possible aggregate state of the world). As there are two ${ }^{3}$ types of agents there would be 44 types of mutual insurance contracts plus the eleven Arrow securities and the bond. As each mutual insurance contract is indexed by the agent who receives the contract, in practice there would be 220 actual contracts traded, plus the Arrow securities and the bond. Due to the large number of possible contracts and the lack of sufficient space we do not describe them here further. Unfortunately, CCW do not give us general guidance as to what kind of insurance demand agents would have. On the other hand, Smith and Mayers(1983) tell us that the presence of other assets will affect insurance demand although it is not clear in which way or even if agents will over- or under-insure.

\footnotetext{
${ }^{1}$ The loading on an insurance contract is given by $\nu_{i}=\left(S_{i}-p_{i} L\right) / p_{i} L$.

${ }^{2} \mathrm{~A}$ mutual insurance contract is a contract of the form: "Pay agent $i$ and amount $x$ if she has $y$ accidents and the total number of accidents in the economy is $z$ ".

${ }^{3}$ We want to emphasize that in this example we have chosen to have the same number of types of agents, 2, as types of hazards, 2. To illustrate the differences between our model and that of CCW, we could have allowed all combinations of high and low $\bar{c}_{i}$ and $w_{i}$, and hence four types of agents, and as long as there were only two types of hazards, $k=2$, and hence would not require extra assets.
} 


\subsection{The Dynamic Economy and Equilibrium}

We are interested in studying an economy which has access to continuously open exchanges. In particular, consider the same economy, but include assets and the possibility of trading these assets as information is (continuously) revealed.

We have already included in the description of the economy how information about accidents arrives: accident arrivals have exponential distributions with parameter $\lambda_{i}\left(N^{e}(t)\right)$. Let us expand the probability space to include the realizations of the arrival times, $\tau_{i}$, and denote the new probability space by $\Omega^{\prime}$ and the new states of the world $\omega^{\prime}$. The information arrival is modelled using a marked point process ${ }^{4}, N(t)$. This process $N(t)$ is a vector of counting processes $\left(N_{1}(t), \ldots, N_{n}(t)\right)$ such that $N_{i}\left(t, \omega^{\prime}\right)=1$ if $\tau_{i}\left(\omega^{\prime}\right) \leq t$ and zero otherwise. Using the accident arrival distributions we can naturally extend $P$ to the probability space $\left(\Omega^{\prime}, \mathcal{F}_{1}\right)$, and model the information revelation using the filtration generated by $N, \mathcal{F}_{t}$, where $\mathcal{F}_{1}=\mathcal{B}\left(\Omega^{\prime}\right)$, i.e. the Borel sets over $\Omega^{\prime}$.

As for the objects of trade, securities, these are defined in the standard way as claims on real consumption at the consumption date, $d_{j}$, indexed by $j \in J$ and their price processes, $S_{j}(t)$, are semi-martingales measurable on the filtration $\left(\mathcal{F}_{t}\right)_{t \in[0,1]}$ such that $S_{j}(1)=d_{j}$ almost surely. The space of feasible trading strategies is also standard, $\theta \in \Theta, \Theta$ is the space of $P$-uniformly bounded predictable and measurable processes on $\left(\mathcal{F}_{t}\right)$. Duffie and Huang(1985) establish that the number of assets needed for markets to be complete is equal to the martingale dimension of $\left(\mathcal{F}_{t}\right)$.

Lemma 3.1. The space of martingales on $\left(\Omega^{\prime}, \mathcal{F}_{1},\left(\mathcal{F}_{t}\right)_{t}, P\right)$ has martingale dimension of at most $n$.

In this economy information flows sufficiently smoothly such that the Pareto optimal state-contingent equilibrium allocations can be decentralized by a dynamic asset trading economy with at most $n$ risky assets and a bond. In terms of characterizing agents' trades, Penalva(1999) established that introducing redundant assets (fully diversified contracts) would reduce the number of assets each agent needs to use to attain her optimal consumption allocation. A fully diversified asset is a contract whose dividends depend only on aggregate events, i.e. $d_{j}(1)=f\left(N^{e}(1)\right)$.

LEMma 3.2. Each agent can attain her optimal Walrasian allocation by trading in insurance on herself, $k$ fully diversified contracts and $a$ bond.

${ }^{4}$ For further details on marked point processes see Brémaud(1981). 
This is because agents' optimal net trades can be decomposed into an agent-specific component and an aggregate component. The agentspecific component is taken care off by the insurance contract and the aggregate part is dealt with using the fully diversified portfolios. In the parametrized economy, $k=2$.

Our aim is to characterize insurance trading in that economy and compare it to the results from the static one. In particular, we want to know if agents would purchase full insurance, over- or underinsure. A full insurance contract is an asset that gives agent $i$ a dividend $d_{i}$ of the form: $d_{i}\left(A_{i}\right)=L$ and zero otherwise.

THEOREM 3.1. If agents have access to a full insurance contract and $k$ fully-diversified portfolios, the agent's optimal insurance demand is to purchase full coverage.

The intuition behind this result follows from Lemma 3.2. The agentspecific component of the optimal allocation comes from the agent's endowment and the aggregate component from the agents' optimal consumption. By purchasing full coverage, the agent takes care of the agent-specific component and then uses the remaining wealth to attain the optimal consumption allocation, which depends only on the aggregate component. Consumption is then achieved using the $k$ redundant fully diversified contracts. By allowing trading in these aggregate assets, market prices will decentralize the allocation of risk such that when all uncertainty is revealed markets clear.

This theorem helps explain why such new assets as catastrophe bonds have been introduced in insurance markets: they bonds help reduce transaction costs as agent's purchase full coverage to protect their property against earthquakes; insurance companies issue bonds that promise a premium over the riskless rate of return if there are no earthquakes but if an earthquake happens, those who purchased the bond stand to loose their interest (and risk premium), as well as their invested capital, which is then used to fulfill the obligations with insurees. By raising capital directly, using the bonds, rather than indirectly, using reinsurance companies, insurance companies eliminate an intermediary and the costs associated with that.

Also, the flexibility of the framework we are using allows one to model potentially new securities such as bonds written on expensive epidemic diseases, like AIDS. These bonds could be used by smaller health insurers to raise capital and cover themselves against the large losses associated with the disease. Our model provides equilibrium prices both for insurance and the bonds, and the amount of capital raised should exactly compensate the amount needed at each state of the world. 
Note also that the fully diversified contracts are redundant assets, so that one cannot expect their supply to be zero. One can only expect that the value of assets and liabilities nets out. The traditional definition of a securities' market equilibrium is that of Radner(1972), which requires the net supply of every asset to be zero (our economy is a pure exchange economy). This is is why we have avoided speaking of an equilibrium. An appropriate notion for our context would be:

Definition 3.1. An equilibrium with redundant assets for the economy described above is an array $\left(\left(S_{j}\right)_{j \in J},\left(x_{i}, \theta_{i}\right)_{i \in I}\right)$ of security price expectations, consumption allocations and trading strategies such that:

(1) there exists $r \in \mathbf{R}_{+}$and $Q$ such that

for all $j \in J, S_{j}(t)=E_{Q}\left[d_{j}(1) \mid \mathcal{F}_{t}\right] /(1+r)$,

(2) for all $i \in I,\left(x_{i}, \theta_{i}\right) \in B\left(e_{i}, S_{j}\right)$, and

$U_{i}\left(x_{i}\right) \geq U_{i}\left(x^{\prime}\right)$ for all $\left(x^{\prime}, \theta\right) \in B\left(e_{i}, S_{j}\right)$,

(3) for all $t \in[0,1], \sum_{j} \sum_{i} \theta_{j}^{i}(t) S_{j}(t)=0$.

Note for any equilibrium of this kind, there is an equivalent Radner equilibrium (same $(r, Q)$ and same $\left(x_{i}\right)$ ) with a set of assets whose net supply is zero - that set of assets is the minimal set required for markets to be complete defined by Duffie and Huang(1985).

We can now speak of the equilibrium initial price of insurance, which would be 0.1298 and 0.3886 for agent types A and B respectively. Let the two fully diversified assets have payoffs as defined on Table 2: An asset with random payoffs, $d_{e 1}$, and a bond that pays a $20 \%$ return on its face value if there are no accidents but if there are any accidents it does not pay any interest and $10 \%$ of the invested capital is lost for each accident that occurs.

\begin{tabular}{|c|ccccccccccc|}
\hline$N^{e}(1, \omega)$ & 0 & 1 & 2 & 3 & 4 & 5 & 6 & 7 & 8 & 9 & 10 \\
\hline$d_{e 1}(\omega)$ & 60 & 55 & 49 & 44 & 38 & 31 & 24 & 17 & 9 & 0 & 91 \\
\hline$d_{e 2}(\omega)$ & 12 & 9 & 8 & 7 & 6 & 5 & 4 & 3 & 2 & 1 & 0 \\
\hline
\end{tabular}

TABLE 2.

Fully diversified contracts

The prices of the two aggregate assets is 57.32 and 10.65. Initial equilibrium asset holdings are given on Table 3.

The agents go short (supply) the first risky asset and use the money to purchase insurance, the cat(-astrophe) bond style asset and save. Over time, the agent reacts to news of accidents by rebalancing the riskless asset, the cat bond and the other risky asset. It is clear that the net 


\begin{tabular}{|c|c|c|c|c|}
\hline Agent Type & Insurance Holdings & \multicolumn{2}{|c|}{ Agg Asset 1} & \multicolumn{3}{|c|}{ Agg Asset 2} & Riskless Asset \\
\hline Type A & 1.0000 & -0.0821 & 0.0957 & 3.5579 \\
\hline Type B & 1.0000 & -0.1858 & 0.2154 & 7.9643 \\
\hline
\end{tabular}

TABLE 3.

Initial Asset Holdings

asset holdings are non-zero for any one security, but the aggregate value of asset holdings is zero.

\subsection{Full Insurance in Partial Equilibrium}

In this section we want to analyze further why agents act contrary to the predictions of the static insurance models when purchasing insurance at unfair prices. We find that this result is due to markets being complete and that information flows gradually.

Let us look at the agent's problem for a given set of (not necessarily equilibrium) security prices. First define the agent's budget constraint: for every agent $i$, let $B\left(e_{i}, S_{j}\right)$ denote the set of consumption strategy pairs $(x, \theta)$ such that $\sum_{j} \theta_{j}(0) S_{j}(0)+x(0)=e_{i}(0), x(1)=$ $e_{i}(1)+\sum_{j} \theta_{j}(1) d_{j}(1)$, and the dynamic trading strategy $\theta \in \Theta$. Then, the agent's partial equilibrium problem is maximizing $U_{i}(x)$ by choice of $(x, \theta)$ subject to $(x, \theta) \in B\left(e_{i}, S_{j}\right)$.

The price processes, $S_{j}(t)$, are given and define the set of attainable consumption allocations, $\mathcal{A}: \mathcal{A}$ is the set of consumption allocations, $x$, such that there exists an initial constant endowment $e=(c, 0), c \in \mathbf{R}$, and $x \in B\left(e, S_{j}\right)$. We will assume that markets are complete, i.e. that $\mathcal{A}=\mathbf{R} \times L(\Omega)$. Hence, the optimal problem can translated into a twostage problem:

(i) Solve $\max _{x} U_{i}(x)$ subject to $x(0)+e^{-r} E_{Q^{\prime}}[x(1)]=e_{i}(0)+e^{-r} E_{Q^{\prime}}\left[e_{i}(1)\right]$, where the interest rate $r \in \mathbf{R}_{+}$and the measure $Q^{\prime}$ are obtained from $S_{j}(t)$ by no-arbitrage;

(ii) Find the trading strategy, $\theta$, that will attain $x$.

Varaiya(1975) shows that these two problems are equivalent and we will use his characterization to analyze optimal trading strategies. Consider an alternative set of security prices for our example: increase the relative value of consumption when there are no accidents and reduce that of states with accidents, but retain the representative agent charac- 
terization of prices. In particular, change $\xi$ to $\xi^{\prime}$ as follows:

\begin{tabular}{|c|ccccccccccc|}
\hline$N^{e}(\omega)$ & 0 & 1 & 2 & 3 & 4 & 5 & 6 & 7 & 8 & 9 & 10 \\
\hline$\xi(\omega)$ & 0.980 & 1.020 & 1.059 & 1.098 & 1.137 & 1.176 & 1.216 & 1.255 & 1.294 & 1.333 & $1.373 \mid$ \\
\hline$\xi^{\prime}(\omega)$ & 0.995 & 1.004 & 1.017 & 1.035 & 1.059 & 1.090 & 1.129 & 1.177 & 1.234 & 1.299 & 1.374 \\
\hline
\end{tabular}

This change of measure implies insurance prices and holdings as described in Table 4.

\begin{tabular}{|c|c|c|c|c|}
\hline Agent Type & Insurance Price & Agg Asset 1 & Agg Asset 2 & Riskless Asset \\
\hline Type A & 0.1263 & 57.38 & 10.68 & 1.00 \\
\hline Type B & 0.3787 & 57.38 & 10.68 & 1.00 \\
\hline Asset Holdings & Insurance & Agg Asset 1 & Agg Asset 2 & Riskless Asset \\
\hline Type A & 1.0000 & -0.0255 & 0.0280 & 1.041 \\
\hline Type B & 1.0000 & -0.0645 & 0.0707 & 2.577 \\
\hline
\end{tabular}

TABLE 4.

Partial Equilibrim Example

We see that agents have lower insurance prices (although still unfair) and the price of the other risky assets has increased, reducing their return. As can be seen in Table 5, the overall effect for an agent of type A (and for type B it is similar) the overall price change leads to lower consumption in the no accident state (and hence excess supply) and higher consumption (excess demand) in the accident states.

More importantly for our analysis, agents readjust their non-insurance asset portfolios but maintain full insurance coverage. They reduce their supply of the first risky asset, and invest less in bonds. This increases the relative weight of insurance and reduces the net availability of capital at the loss states, making it impossible to satisfy insurance liabilities and market clearing.

\begin{tabular}{|c|ccccccccccc|}
\hline$N^{e}(1, \omega)$ & 0 & 1 & 2 & 3 & 4 & 5 & 6 & 7 & 8 & 9 & 10 \\
\hline$\xi, x_{A}(\omega)$ & 10.08 & 9.68 & 9.28 & 8.89 & 8.49 & 8.09 & 7.69 & 7.30 & 6.90 & 6.50 & 6.11 \\
\hline$\xi^{\prime}, x_{A}(\omega) \mid$ & 9.92 & 9.83 & 9.70 & 9.52 & 9.28 & 8.96 & 8.57 & 8.08 & 7.50 & 6.85 & 6.09 \\
\hline
\end{tabular}

TABLE 5.

Partial Equilibrium Consumption

This is quite surprising; one would have expected agents to increase their demand for cheaper insurance. The following theorem shows that 
we should not expect agents to change their demand for insurance for certain kinds of price changes.

TheOREM 3.2. Suppose each agent has access to a full insurance contract such that markets are complete and that the implied martingale measure $Q^{\prime}$ is defined by the Radon-Nikodym derivative $\mathrm{d} Q^{\prime} / \mathrm{d} P=\xi^{\prime}\left(N^{e}(1)\right)$. Suppose further that there is a complete set of (redundant) fully diversified contracts and a bond. Then the agent's optimal trading strategy can be described as: buy and hold full coverage and dynamically trade the diversified contracts and the bond.

The full insurance property we observed in Theorem 3.1 extends to the partial equilibrium optimal asset demand problem and leads to two interesting corollaries which require no proof:

Corollary 3.1. Under the conditions of Theorem 3.2, agents can optimally purchase full coverage independently of whether the insurance contract is fairly priced or not.

COROLlary 3.2. Under the conditions of Theorem 3.2, all agent's optimal demand for insurance will be the same, regardless of the correlation of its price with that of the fully diversified assets in her portfolio.

This is clearly counter to classical results. The reason has to do with two things: one, information is revealed nicely and two, there is an (effectively) full set of assets. The more intuitive effect is that of complete markets. Smith and Mayers(1983) consider a context where markets are incomplete. Assets play a dual role: they serve as wealth transfer instruments (accross time and states of the world) and they define the incompleteness in markets, the restrictions on how wealth can be transferred accross states. These cross state restrictions distort insurance demand in as far as the insurance contract helps aleviate these restrictions. As we consider complete markets, assets become simple wealth transfer instruments and the role of insurance as an agent-specific transfer can be separated from the role of transferring consumption accross states of the world with different aggregate consumption.

The gradual revelation of information plays a key role in furthering the separation of agent-specific trading and aggregate diversification. Dynamic trading allows the agent to react to the gradual revelation of information. In as far as that information separates agent-specific news from aggregate news, the agent will be able to reflect that distinction in her trading behaviour. Allowing for the hazard to depend on 
the aggregate number of accidents still respects that separation. The agent can eliminate her agent-specific risk by purchasing a full insurance contract and then adjust her wealth holdings in response to aggregate relative scarcity as reflected in the prices and returns of fully diversified portfolios.

In our example, the announcement of a loss to an agent implies the same loss for the economy, $L$, at date one. But, because this loss implies one less agent at risk, this will affect the probabilities over future losses depending on whether the agent was of type A or type B. The probability that the total number of accident is two, conditional on the first announcement of an accident, depends on whether there are 4 agents of type A and 5 of type B still at risk, or 5 of type A and 4 of type B. This is why in our example $k=2$ and we needed two fully diversified contracts. Two is the right number of assets to have enough flexibility to allocate our wealth optimally depending on which type of agent has an accident, and fully diversified assets are the right kind needed to deal with aggregate risk. Even if the agent's hazard depends on the aggregate number of losses would we still need only two assets.

We have seen why agents prefer to purchase full insurance in this economy that allows continuously traded assets. Positive insurance loadings are a natural outcome of the decentralization process as they are needed to compensate the providers of capital for aggregate uncertainty. Agents need to be encouraged to acquire net negative aggregate positions in states of the world in which there are aggregate losses and the only way to do so is by offering a positive return on the aggregate assets which compensates them for that risk. This way, the insurance market's assets and liabilities net out: they use premium income to compensate capital providers when losses are few so that they can use that to compensate insurees when losses are high.

\section{ASYMMETRIC INFORMATION AND RESTRICTED TRADING}

We want to use this framework to study the problem of asymmetric information. In particular, we want to consider the problem of how to regulate genetic testing in insurance markets (specially life and health insurance). Our model is that of an exchange economy and should be considered as a first step toward a more extensive study of the problem. The main characteristics of the problem we want to consider here is that information acquisition (the test) is costly to do systematically but in some cases it is obtained for free. The existence of the possibility of doing and interpreting these tests correctly can lead to problems of asymmetric information in insurance markets. This asymmetric information can be 
on the side of the insuree, who knows the results of the test and refrains from disclosing it (whether for legal or privacy issues). It can also be on the part of the insurer who has all details of the test and its implications due to better access to data and expertise in interpreting the information. We will continue to assume that all agents are price takers and the Walrasian auctioneer plays the role of the insurance industry in setting competitive prices.

\subsection{The Problem and Possible Solutions}

There has been a tendency for insurance prices to remain relatively stable and agents to remain with their insurance company for long periods of time. This trend is changing and we are observing more activity both by the insurer (changing rates and adjusting coverage) and the insured (switching companies). These changes are most probably due to private information. We want to know what this means for the stability and future properties of insurance markets, and interpret it in the context of genetic testing.

The problem we want to consider is what happens if there is a method for obtaining information which reveals whether an agent is more or less at risk than previously thought and that information is not shared by all parties (the agent and the insurance companies with which she works). In many countries, this situation arises as privacy laws protect those who do not wish to reveal the results from genetic tests, and as insurance companies who have genetic information (via blood tests or other tests) use it to obstruct or deny coverage.

The context we are considering is equivalent to that considered by Grossman and Stiglitz(1980, GS) and they conclude that informationally efficient markets are impossible with costly information acquisition. The alternative left is informationally inefficient markets, i.e. for prices not to reflect all available information. The question we try to answer is whether informationally inefficient markets are possible and allocationally efficient. We base our analysis on the initial insights of Milgrom and Stokey(1982).

Our model illustrates the problem quite clearly: suppose the insurance company pays for the genetic test on agent $i$ and does not reveal the result of the test. If the test makes agent $i$ more at risk then the insurance company can do two things: hike up the price of insurance or cancel the coverage. If the price goes up, nothing happens: the agent already holds the insurance contract and its value goes up but she continues to want to hold the same amount of coverage (if the new price fully reveals the information); the insurance company sees its liabilities increase. To compensate, the insurance company needs to test all its insurees which will reveal that the increased liability from $i$ is balanced out by all the 
others and the balance sheet is fine. The gain for the insurance company is zero, but it has spent all this money testing insurees, so adjusting prices and doing all the tests is not worthwhile (hence, not an equilibrium outcome).

If, on the other hand, the company denies coverage, then the agent will not find another insurance company which will take her - lost coverage implies a risk too high for current rates. But this means that the agent wants to purchase insurance at current rates but no one will supply it again, we find ourselves out of equilibrium (demand does not equal supply). The other insurance companies will only accept her if she pays a higher premium, but the agent, when she lost coverage, was not compensated for the cancellation of the coverage at the higher post-test rates, so that she will need to readjust her wealth allocation and break the initial equilibrium. The economy is left with two possibilities for equilibrium: either include the price of the test in the initial insurance contract together with a guarantee of coverage (this is equivalent to Tabarrok's "genetic insurance" - see Tabarrok(1994)) or not do the test at all. The first case, if possible, is Pareto dominated by the second. But not doing the test is only possible if the tests are never done. If for some reason (say via a free test prior to blood donation, for scientific purposes, ...) the test information can be obtained for free then the second alternative disappears as an equilibrium.

An alternative would be to consider suspending trade. According to Milgrom and Stokey(1982) if agents can negotiate ex ante efficient contracts then the new information will not generate any trade so the suspension of trade does not add inefficiencies. The problem is that if one does not allow trading we return to the static insurance economies and we will find that market completeness requires too many or too complex assets.

Since GS's paper there have been a number of alternative models of general equilibrium with asymmetric information addressing the existence issue in different ways ${ }^{5}$. Some have included noise in price setting which would translate to: the results of the tests on increased coverage are not fully deducible from prices (Hellwig (1980), Diamond and Verecchia(1981), Admati(1985)); others have added imperfect competition in price setting where agents are aware of the effect of their asset demands on prices (Kyle(1985, 1989), Bhattacharya and Spiegel(1991), Holden and Subrahmanyam(1992)); and yet another group has considered different non-linear pricing schedules to ensure equilibria exist (Prescott and Townsend(1984), Bisin and Gottardi(1998, 2000)). We retain price-

\footnotetext{
${ }^{5}$ There is also a very large literature on asymmetric information in game theory and with partial equilibrium. Some particularly relevant papers are Doherty and Thistle(1996) and Strohmenger and Wambach(2000).
} 
taking behaviour, exclude additional sources of uncertainty (such as noise traders) and maintain linear prices (whenever trading is permitted). Nevertheless, our model is closest to those with minimal non-linearities in prices by Bisin and Gottardi(1998, 2000)

\subsection{Our Approach}

The way asymmetric information can be incorporated into our model is by adding signals which are received between consumption dates. If we just look at the results of the previous section, an interesting and striking feature of insurance trading behaviour is that despite the revelation of information (how many agents have suffered accidents, ...) agents do not wish to change their insurance holdings. So any agent who wants to change her insurance holdings immediately signals that she has some information not known to the insurer. Similarly, any attempts to reduce coverage, add restrictions on existing coverage, or add unexpected charges on the part of the insurer also reveals that the insurer has information not known to the insured.

Not doing the test at all is the non-informationally efficient price equilibrium and corresponds to the "full information" model in the previous sections - full information in the sense of common priors, common knowledge and full public disclosure of news. It is an equilibrium as long as the signal cannot (or is too expensive) to appear. Given historical insurance trading behaviour one could argue that in the past there was some kind of formal or informal institutional arrangement that kept common knowledge and public (lack of) information, and that arrangement has broken down - presumably because information acquisition has become cheaper or easier. As we will see, being uninformed may not have been inefficient and the increased flow of information may be making the insurance market unstable - Hirschleifer(1971) has already pointed out that more information is not necessarily better.

Let us introduce asymmetric information into the model formally. Let $y_{i}(t)$ be a random process, which we will call a signal on agent $i$, and let $\tilde{y}(t)$ denote the vector of signals on all the agents. Rather than introducing complex notation to describe the filtration that incorporates $\tilde{y}$, we will just add a second argument to the conditional expectation operator and extend the terminal $\sigma$-algebra from $\mathcal{F}$ to $\mathcal{F} \vee \mathcal{F}_{Y}, \mathcal{F}_{Y}=$ $\sigma(\tilde{y}(s), s \leq 1)$. The interpretation of the notation should be obvious. Let the vector of signals have the following properties:

No initial signal There are no signals at the start date: $\tilde{y}=(0,0, \ldots, 0)$.

Individual Non-neutrality The signals contain information: there exists $t \in(0,1)$ and $A \in \mathcal{F}, \mathbf{1}_{A} \in L_{\infty}\left(\Omega, \mathcal{F} \vee \mathcal{F}_{Y}, P\right)$, such that $E_{P}\left[\mathbf{1}_{A} \mid \mathcal{F}_{t}, \tilde{y}(t)\right] \neq$ $E_{P}\left[\mathbf{1}_{A} \mid \mathcal{F}_{t}\right]$ 
Aggregate Neutrality The signals do not affect the distribution of the aggregate endowment process: for any real valued function, $f$, $E_{P}\left[f(w(1)) \mid \mathcal{F}_{w, t}, \tilde{y}(t)\right]=E_{P}\left[f(w(1)) \mid \mathcal{F}_{w, t}\right]$.

To illustrate these definitions return to the example we used in the previous section. Let all the parameters be the same except change the hazards, $\lambda_{i}$. Impose that all hazards are zero up to date $t=1 / 2$. Then, imagine a lottery that randomly assigns five agents to have $\lambda_{i}=0.051$ and the other five $\lambda_{i}=0.156$. Each agent finds out whether she has a high or low hazard, but no one else does. This signal clearly satisfies the individual non-neutrality as the probability of a loss changes for each agent. In order to satisfy the second assumption we cannot let agent's risks be independent otherwise the probability of future accidents depends on whether the last accident was of an agent with high or low hazard. We let accidents come in pairs: when there is an announcement, both an agent with high and an agent with low hazard have an accident. This ensures that private information does not have aggregate effects. Then the question is what is the best way to deal with this asymmetric information.

Clearly, the assumption of aggregate neutrality of signals is a strong one. We wish to concentrate on signals that have only a purely agentspecific content and with a finite number of agents one needs a strong assumption such as this one for signals to have purely agent-specific information. We would not need this assumption if we had a continuum of agents but then again assuming that there are as many agents as real numbers in an interval is also a strong assumption. Clearly aggregate neutrality is only valid when there is a sufficiently large number of agents that knowing that a single agent has a higher or lower hazard has a negligible effect on one's perception of the value of assets whose payoffs depend on economy-wide variables.

We have seen above that the economy without the signal has an equilibrium, but that equilibrium cannot be implemented if some agents receive the signals. An agent with a signal on himself that is not publicly known wants to alter his insurance trades if we keep the prices from the nosignals economy. But, as he is the only one with this information, the rest of the market does not want to alter their allocations to accomodate his change in demand (at those prices). If prices reveal all the information, agents will maintain the same insurance trades and attain the same allocations:

THEOREM 4.1. If in the economy of Theorem 3.1, the insurance contracts available for each agent are full coverage insurance contracts, $d_{i}(1)=$ 
$\mathbf{1}_{A_{i}} L$, and the price of agent $i$ 's insurance contract at date $t \in[0,1)$ is:

$$
S_{i}(t)=\frac{E_{Q}\left[L \mid \mathcal{F}_{t}, Y_{t}\right]}{1+r}
$$

where $\mathrm{d} Q / \mathrm{d} P$ and $r$ are the same as in Theorem 3.1, then there exists a redundant asset equilibrium where agents can attain the same Pareto optimal Walrasian equilibrium allocations using the same strategies as those in Theorem 3.1.

But, this equilibrium requires that everyone has genetic tests whose results are sufficiently public to be incorporated into prices. Trivially, if those tests require some expense, $c>0$ for the whole economy, the equilibrium with full information plus the cost is Pareto dominated by the equilibrium of Theorem 3.1, which attains the same consumption allocations without incurring the loss of $c$.

The issue now is what does the government do knowing that genetic tests are costly and aware that some genetic tests are being done. It cannot impose a non-informationally efficient equilibrium because some agents have or will have access to genetic information costlessly - hence destroying the equilibrium. It could impose the full information equilibrium at considerable cost for the economy. It could suspend all dynamic trading, but this would eliminate dynamic trading opportunities and complicate the existence of complete markets and hence the attainability of efficient allocations. Another alternative, which is the most common current policy, is to restrict trading but only partially: it can allow agents to acquire genetic information but not allow that information to enter into insurance transactions. The last option has the problem, suggested by insurance practitioners, that even if that information cannot officially enter transactions, somehow it will eventually leak into those transactions, and "A consent law which hid information de jure but not de facto could be worse than no law at all" (Tabarrok, 1994). Another way to implement no intermediate trading in insurance is to rule out new insurance transactions (leaving global asset market transactions as before).

TheOREM 4.2. If in the economy of Theorem 3.1,

1.the insurance contracts available for each agent are full coverage insurance contracts, $d_{i}(1)=\mathbf{1}_{A_{i}} L$;

2.agents are restricted to buy-and-hold strategies on these insurance contracts, $\theta_{i, i}(0) \in \mathbf{R}, \forall t \in(0,1], \theta_{i, i}(t)=\theta_{i, i}(0) ;$ and,

3.there is a signal vector process, $\tilde{y}(t)$, that satisfies the individual nonneutrality and aggregate neutrality properties, 
then there exists a redundant asset equilibrium where agents can attain their Pareto optimal Walrasian equilibrium allocations independently of the identity and the number of agents who observe $\tilde{y}(t)$. The price process of the fully diversified portfolio and the optimal trading strategies will be those of Theorem 3.1.

Corollary 4.1. In the economy of Theorem 4.2, if for all $i \in I$, we allow agent $i$ to observe the two processes $\left(N(t), y_{i}(t)\right)$ then there exists a redundant asset equilibrium such that for all $i \in\{1, \ldots, n\}$, agent $i$ can attain her optimal Walrasian equilibrium allocation.

The intuition is quite straight-forward. The economy at date zero looks just like the perfect information economy of Theorem 3.1 and the same optimal consumption allocations and trading is feasible. If we keep the same price processes for all assets, then allowing any agent to observe any number of the signals then, by the aggregate neutrality of signals, she would only want to change individual insurance-related trades, but those are ruled out by construction. The agent-specifity of the information allows to partially suspend trade in the contracts that were ex-ante efficient (insurance) and dynamically trade to optimally diversify in the presence on new aggregate information (trading in stock markets). As the consumption allocation was optimal among feasible consumption allocations that only depended on the aggregate endowment, the current allocation is optimal and within the span of the aggregate assets.

This result implies that non-insurance prices can adjust to incoming accident information (because of their aggregate information content) and private insurance prices can only change according to the same information as before genetic testing: accident history and ex-ante risk type (the properties of the hazard function). Agents are then allowed to choose their insurance allocations at the beginning and are forced to hold them till the end. This is equivalent to enforcing long-term insurance contracts, negotiated prior to the possibility of attaining private information.

As Hirschleifer anticipated, the early realization of uncertainty can only eliminate risk-sharing possibilities. Informationally efficient markets are not valuable per se. The introduction of genetic testing will only lead to an arms race of testing that will eventually just readjust prices without improving the welfare of future generations and possibly raising the costs of insurance.

If on the other hand we were allowing for production of some sort, where information on the realization of accidents affects the optimal al- 
location of resources, then there may be some value for this information and that value has to weighted relative to the cost of acquiring the information. In terms of genetic testing, there is no need to spend resources on tests if those test only serve to increase the insurance rates for some agents and lower those of others. If the tests help coordinate resources, say by constructing a more efficient network of specialized hospitals, efficient preventive treatment, ... then the costs of testing have to be weighed against the benefits. If the gains are higher then the tests can be done and the hospitals constructed. But there is no reason why this information has to affect the price of individual insurance - the risk is optimally shared before the tests are out.

\section{GENERALIZATIONS OF THE MODEL}

We have seen above that insurance demand becomes independent of other assets demands if there are enough other assets. Above one needed $k$ assets to deal with the $k$ different classes of hazard functions. One question to ask is whether rather than have agents with different hazards they could have different magnitudes of losses. In general the answer is yes, $k$ is determined the number of risks that describe the aggregate endowment process and one can allow for different hazards, different properties of hazards (add Markov processes on which hazards depends, ...). A more general study of the kinds of risks that could be treated in this framework and the number and types of assets needed to deal with them has been studied by Penalva(1999).

This generalizations apply also to the full insurance result of Theorem 3.1. By extending the notion of a full insurance contract as one that compensates one-for-one all losses suffered by the agent, Theorem 3.1 can be generalized in ways that will take us too far from the aim of this paper but which include multiple loss magnitudes, different risks, etc. The most obvious extension is to consider the possibility of more than one loss per agent. By extending the notion of full insurance to cover all the losses we can apply the arguments in the proof of Theorem 3.1 to show that the same result holds.

As for the efficiency properties of suspending insurance trade, as long as we retain the same properties of the signals, the extensions are totally analogous. The only thing to recall is that the assumption of aggregate neutrality is only meaningful in contexts were individual information has little aggregate content, such as when there are many agents with independent or weakly correlated risks. 


\section{CONCLUSION}

We have shown that an economy with insurance markets and with other continuously traded assets behaves quite differently than the ones in classic insurance theory. In particular, we have found that agents buy full insurance even if prices are unfair and there are assets with payoffs correlated with that of insurance contracts. In terms of insurance pricing, insurance companies, as intermediaries between the individual at risk and the global asset market, should charge positive loadings in as far as those loadings are required to compensate asset markets for the aggregate risk they generate. Despite those loadings agents should be able to attain efficient consumption levels. The possibility of efficient insurance purchases in such a context is primarily due to the optimality of eliminating agent-specific risk and the possibilities of separating insurance trading from the trading on fully diversified portfolios permitted by the way information is revealed over time.

We have also seen that information can generate problems for the existence of markets and that the revelation of information is not necessarily welfare enhancing (the well-known Hirschleifer effect). In particular, it is impossible for markets to be informationally efficient if information acquisition is costly and the presence of information can destabilize noninformationally efficient ones. Hence, the suspension of informational trades (implicit in Milgrom and Stokey, 1982), which can be considered the equivalent of restricting insurance transactions not to be based on genetic information, stabilizes the market and insures efficient insurance purchases. If such legal measures are not sufficient, they could be implemented by imposing long-term health contracts, based on pre-test information. Changes of insurers could be done at prices established at an industry level via consensus or government mandate. These prices would incorporate all appropriate public data, as is done under risk adjustment policies. As for the diversification of risks accross insurance companies, this can be done ensuring them access to dynamic secondary markets that aggregate and diversify these risks effectively. As long as the private information has no aggregate effects, such markets need not be concerned about it.

Our model has concentrated on the purely informational effect of genetic testing. The cases in which genetic testing has real efficiency effects in terms of production of resources, such as optimal allocation of specialized hospitals or equipment, and the existence of efficient post-test therapies, have not been considered explicitly. Nevertheless, it is possible to analyze those effects separately and compare them with the cost of the tests, while isolating insurance transactions from this information. The debate on the role of genetic testing is a rich and complex one and 
we have only addressed a single issue and on a limited basis. We hope that this paper serves as a contribution to clarify this debate.

\section{REFERENCES}

1. Admati, Anat R. (1985): "A Noisy Rational Expectations Equilibrium for Multi-asset Securities Markets," Econometrica, 53(3), 629657.

2. Bhattacharya, Utpal and Matthew Spiegel (1991) "Insiders, Outsiders, and Market Breakdowns," Review of Financial Studies, $4(2), 255-282$.

3. Bisin, Alberto ANd Piero Gottardi (1998) "Competitive Equilibria with Asymmetric Information: Existence with Entry Fees", mimeo - available at http://www.econ.nyu.edu/user/bisina/EFEE2.PDF.

4. Bisin, Alberto And Piero Gottardi (2000) "Decentralizing Incentive Efficient Allocations of Economies with Adverse Selection", mimeo - available at http://www.econ.nyu.edu/user/bisina/RSNLL.PDF.

5. Borch, K. (1990): Economics of Insurance. Edited and completed by Knut K. Aase and Agnar Sandmo, North-Holland.

6. Brémaud, P. (1981): Point Processes and Queues: Martingale Dynamics., Springer-Verlag. New York.

7. Cass, David, Graciela Chichilnisky and Ho-Mou Wu (1996): "Individual Risk and Mutual Insurance," Econometrica, 64, 333-341.

8. Diamond, Douglas W. and Robert E. Verecchia (1981): "Information Aggregation in a Noisy Rational Expectations Economy," Journal of Financial Economics, 9, 221-235.

9. Doherty, Neil A. And Paul D. Thistle (1996): "Adverse selection with endogenous information in insurance markets," Journal of Public Economics, 63 , 83-102.

10. Duffie, D. And C. Huang (1985): "Implementing Arrow-Debreu equilibria by continuous trading of few long-lived securities," Econometrica, 53, 1337-1356.

11. Grossman, Sanford J., And Joseph Stiglitz (1980): "On the Impossibility of Informationally Efficient Markets," The American Economic Review, 70(3), 393-405.

12. Hellwig, Martin F. (1980) "On the Aggregation of Information in Competitive Markets," Journal of Economic Theory, 22, 477-498. 
13. Hirschleifer, J. (1971) "The Private and Social Value of Information and the Reward to Incentive Activity," American Economic Review, 61, 561-574.

14. Holden, Craig W. And Avanidhar Subrahmanyam (1992) "Long-Lived Private Information and Imperfect Competition," The Journal of Finance, XLVII(1), 247-270.

15. Huang, Chi-Fu (1987) "An Intertemporal General Equilibrium Asset Pricing Model: The Case of Diffusion Information," Econometrica, 55(1), 117-142.

16. Kyle, Albert S. (1985) "Continuous Auctions and Insider Trading," Econometrica, 53(6), 1315-1335.

17. (1989) "Informed Speculation with Imperfect Competition," Review of Economic Studies, 56, 317-356.

18. Last, G. And A. Brandt (1995): Marked Point Processes on the Real Line: The Dynamic Approach., Springer-Verlag. New York.

19. Milgrom, P. AND N. Stokey (1982): "Information, Trade and Common Knowledge," Journal of Economic Theory, 26, 17-27.

20. Penalva Zuasti, Jose S. (1999) Insurance with Frequent Trading: A Dynamic Analysis of Efficient Insurance Markets, Universitat Pompeu Fabra, Department of Economics and Business Working Paper 460. Available at http://www.econ.upf.es/deehome/what/wpapers/postscripts/460.pdf.

21. Prescott, Edward C. And Robert M. Townsend (1984) "Pareto Optima and Competitive Equilibria with Adverse Selection and Moral Hazard," Econometrica, 52(1), 21-45.

22. RADNER, R. (1972): "Existence of equilibrium of plans, prices and price expectations in a sequence of markets." Econometrica, 40, 13651379.

23. Smith, C.W. AND D. Mayers (1983): "The Interdependence of Individual Portfolio Decisions and the Demand for Insurance," Journal of Political Economy, 91(2), 304-311.

24. Strohmenger, R. And A. Wambach (2000): "Adverse Selection and categorical discrimination in the health insurance markets: the effects of genetic tests," Journal of Health Economics, 19, 197-218.

25. Tabarrok, Alexander (1994): "Genetic Testing: an Economic and Contractarian Analysis." Journal of Health Economics, 13, 75-91.

26. VARAIYA, P. (1975) The martingale theory of jump processes, IEEE Trans. Automatic Control, AC-20, 34-41.

27. Wilson, Robert (1968): "The Theory of Syndicates," Econometrica, 36, 119-132. 


\section{APPENDIX}

\subsection{Proof of Lemma 3.1}

This result follows directly from the martingale representation theorem (see Last and Brandt(1991) for a general version and proof of this theorem). The filtration $\left(\mathcal{F}_{t}\right)_{t \in[0,1]}$ is by construction the filtration generated by the marked point process $N(t)$. Define the $\left(\left(\mathcal{F}_{t}\right), P\right)$-martingale process $M(t)=N(t)-\int_{0}^{t} \lambda(s) \mathrm{d} s$, where $\lambda(s)$ is the vector of hazards $\left(\lambda_{1}, \ldots, \lambda_{n}\right)$. As the dimension of this vector is $n$ and (by the martingale representation theorem) for every $\left(\left(\mathcal{F}_{t}\right), P\right)$-martingale, $X(t)$, there is a predictable process $h(t)$ such that $X(t)$ can be represented in integral form as:

$$
X(t)=X(0)+\int_{0}^{t} h(s) \mathrm{d} M(s)
$$

then the dimension of the space of $\left(\left(\mathcal{F}_{t}\right), P\right)$-martingales is at most $n$.

\subsection{Lemma 3.2}

The proof of this Lemma is somewhat involved so we will only run through the main steps. For a detailed proof see Penalva(1999).

Duffie and Huang(1985, DH) demonstrate how to decentralize a Walrasian equilibrium as an equilibrium in security trading (a Radner equilibrium). Their demonstration shows that for each agent there is a strategy that will allow her to attain her optimal consumption. That strategy is obtained by replicating a martingale, which we shall refer to as the wealth martingale, $X_{i}(t)$.

Penalva(1999) takes that martingale and shows that, under the conditions of risk considered in our model, it can be decomposed into an agent-specific component and an aggregate component. With this decompositions he proves that $X_{i}(t)$ is in the span of $k$ fully diversified portfolios and the insurance contract, and hence those assets (plus the riskless bond) are sufficient for $i$ to attain her optimal consumption allocation.

\subsection{Proof of Theorem 3.1}

In order to prove this result we need some auxiliary lemmas.

Lemma 7.1. Given a Walrasian equilibrium pair $\left(\pi,\left(x_{i}\right)_{i \in I}\right)$ there exists a measure $Q$ and an interest rate $r$ such that for all feasible consumption allocations, $y, \pi(y)=y(0)+E_{Q}[y(1) /(1+r)]$, where $E_{Q}[\cdot]$ is the expectation with respect to the measure $Q$. Also, $\mathrm{d} Q / \mathrm{d} P=\xi(e(1))$ and for all $i \in I$ there exists $g_{i}: \mathbf{R} \rightarrow \mathbf{R}$ such that $x_{i}(1)=g_{i}(e(1))$. 
The measure and the interest rate correspond to a renormalization of the price vector. The properties of $\xi$ and $x_{i}$ arise from the existence of a representative agent characterization of $\left(\pi,\left(x_{i}\right)\right)$ (see Huang(1985) for a general version of this statement).

Let us introduce a new vector process, $\tilde{N}$. Define it as

$$
\tilde{N}(t)=\left(\begin{array}{c}
\sum_{i \in I_{1}} N_{i}(t) \\
\cdots \\
\sum_{i \in I_{k}} N_{i}(t)
\end{array}\right) .
$$

Let $\left(\mathcal{G}_{t}\right)_{t \in[0,1]}$ be the filtration generated by $\tilde{N}(t)$. Naturally, for all $t$, $\mathcal{G}_{t} \subset \mathcal{F}_{t}$, and $\tilde{N}(t)$ admits the intensity process $\tilde{\lambda}(t)$, where

$$
\tilde{\lambda}(t)=\left(\begin{array}{c}
\sum_{i \in I_{1}} \lambda_{i}(t) \\
\cdots \\
\sum_{i \in I_{k}} \lambda_{i}(t)
\end{array}\right) .
$$

Let $\tau^{j}(t)$ denote the time of the last jump of the process $\sum_{i \in I_{j}} N_{i}(t)$ up to time $t$. As for all $i=1, \ldots, n, \lambda_{i}(t)=\lambda_{i}\left(N^{e}(t)\right)$, then for all $j=1, \ldots, k, \sum_{i \in I_{1}} \lambda_{i}(t)=\tilde{\lambda}_{j}\left(N^{e}(t), \sum_{i \in I_{j}} N_{i}\left(\tau^{j}\right)\right)$, so that $\tilde{\lambda}(t)$ is $\mathcal{G}_{t^{-}}$ measurable and predictable.

Lemma 7.2. For all $t \in[0,1], f: \mathbf{R} \rightarrow \mathbf{R}$, the $\left(\mathcal{F}_{t}, P\right)$-martingale, $X(t) \equiv E_{P}\left[f\left(N^{e}(1)\right) \mid \mathcal{F}_{t}\right]$ is a $\left(\mathcal{G}_{t}, P\right)$-martingale.

Proof: For any $n \in \mathbf{N}$, let $A_{n} \equiv\left\{N^{e}(1)=n\right\}$. Clearly $\left(A_{n}\right)$ is the minimal partition that generates $\sigma\left(N^{e}(1)\right)$. As $\sigma\left(N^{e}(1)\right) \subset \mathcal{G}_{1}$, $f\left(N^{e}(1)\right) \in \mathcal{G}_{1}$. The rest follows from the properties of $\tilde{N}, \tilde{\lambda}$, and martingales.

Also, by applying the martingale representation theorem as we did in Lemma 1 , the dimension of the space of $\left(\left(\mathcal{G}_{t}\right), P\right)$-martingales is at most $k$.

Proof: [Theorem 3.1] Following the same arguments as were used in Lemma 2, all we need to show is that $X_{i}(t)$ is spanned by a full insurance contract and the $k$ fully diversified insurance contracts.

Recall the definition of $X_{i}(t)$ in $\mathrm{DH}$ :

$$
\begin{aligned}
X_{i}(t) & \equiv E_{Q}\left[\left(x_{i}(0)-e_{i}(0)\right)+\frac{\left(x_{i}(1)-e_{i}(1)\right)}{(1+r)} \mid \mathcal{F}_{t}\right]-E_{Q}\left[\left(x_{i}(0)-e_{i}(0)\right)+\frac{\left(x_{i}(1)-e_{i}(1)\right.}{(1+r)}\right] \\
& =E_{Q}\left[\frac{\left(x_{i}(1)-e_{i}(1)\right)}{(1+r)} \mid \mathcal{F}_{t}\right]-C_{i},
\end{aligned}
$$


where $C_{i} \in \mathbf{R}$. For the purposes of analyzing the martingale properties of $X_{i}(t)$ we can ignore $C_{i}$.

The equilibrium price of insurance is $S_{i}(t)=Q\left(A_{i}, t\right) L /(1+r)$, where $Q\left(A_{i}, t\right)$ is the probability agent $i$ suffers a loss (event $A_{i}$ ) given the information up to time $t$, then for $t \in[0,1]$,

$$
\begin{aligned}
X_{i}(t)-S_{i}(t) & =E_{Q}\left[\frac{\left(x_{i}(1)-e_{i}(1)\right)}{(1+r)} \mid \mathcal{F}_{t}\right]-S_{i}(t) \\
& =E_{Q}\left[\frac{x_{i}(1)-w_{i}+\mathbf{1}_{A_{i}} L}{(1+r)} \mid \mathcal{F}_{t}\right]-\frac{Q\left(A_{i}, t\right) L}{1+r} \\
(1+r) X_{i}(t)-S_{i}(t) & =E_{Q}\left[x_{i} \mid \mathcal{F}_{t}\right]-w_{i}+E_{Q}\left[\mathbf{1}_{A_{i}} \mid \mathcal{F}_{t}\right] L-Q\left(A_{i}, t\right) L \\
& =E_{Q}\left[x_{i} \mid \mathcal{F}_{t}\right]-w_{i}+Q\left(A_{i}, t\right) L-Q\left(A_{i}, t\right) L \\
X_{i}(t)-S_{i}(t) & =\frac{E_{Q}\left[x_{i} \mid \mathcal{F}_{t}\right]-w_{i}}{(1+r)}
\end{aligned}
$$

The agents problem is now to replicate $X_{i}(t)-S_{i}(t)$. From Lemma 7.1:

$$
X_{i}(t)-S_{i}(t)=\frac{E_{P}\left[\xi\left(N^{e}(1)\right) g_{i}\left(N^{e}(1)\right) \mid \mathcal{F}_{t}\right]-w_{i}}{1+r}
$$

Which implies that $X_{i}(t)-S_{i}(t) \in \mathcal{G}_{t}$ by Lemma 7.2. Returning to the formal full definition of $X_{i}(t), X_{i}(t)-S_{i}(t)$ is an $\left(\mathcal{F}_{t}, Q\right)$-martingale as both $X_{i}(t)$ and $S_{i}(t)$ are so. As the dimension of the space of $\left(\left(\mathcal{G}_{t}\right), Q\right)$ martingales is the same as that of the $\left(\left(\mathcal{G}_{t}\right), P\right)$-martingales and that of the latter is $k$, one needs at most $k$ assets to replicate $X_{i}(t)-S_{i}(t)$. As one can construct those assets by using $d_{j}(1)=f_{j}\left(N^{e}(1)\right)$, those assets can be fully diversified portfolios.

\subsection{Proof of Theorem 3.2}

Proof: By the proof of Theorem 3.1, it should be clear that all we have to show is that given $\xi^{\prime}\left(N^{e}(1)\right)$, the optimal consumption allocation for agent $i, x^{*}$ is of the form $\left(x_{0}^{*}, x_{1}^{*}\left(N^{e}(1)\right)\right.$, or rather that there exists $h_{i}: \mathbf{R} \rightarrow \mathbf{R}$, such that $x^{*}(1)=h_{i}\left(N^{e}(1)\right)$.

This is relatively straight forward to demonstrate by contradiction: suppose that the optimal allocation is not of the form $x_{1}^{*}=h_{i}\left(N^{e}(1)\right)$. This implies that there is at least one $n \in \mathbf{N}$ such that $x^{*}(1) \mathbf{1}_{A_{n}} \neq c_{n} \mathbf{1}_{A_{n}}$ where $c_{n} \in \mathbf{R}$. (recall $A_{n}=\left\{N^{e}(1)=n\right\}$ ). Consider the alternative allocation $x^{\prime}$ such that $x^{\prime}(0)=x^{*}(0)$ and $x^{\prime}(1)=\mathbf{1}_{\Omega \backslash A_{n}} x^{*}(1)+$ 
$\mathbf{1}_{A_{n}} E_{P}\left[x^{*}(1) \mathbf{1}_{A_{n}}\right]$. Let $\bar{x}=E_{P}\left[x^{*}(1) \mathbf{1}_{A_{n}}\right]$. If we compare the expected utilities of both consumption bundles we obtain

$$
\begin{aligned}
U_{i}\left(x^{\prime}\right) & =u_{i}\left(x^{\prime}(0)\right)+\beta_{i} E_{P}\left[u_{i}\left(x^{\prime}(1)\right)\right] \\
& =u_{i}\left(x^{*}(0)\right)+\beta_{i}\left(E_{P}\left[u_{i}\left(x^{*}(1)\right) \mid \neg A_{n}\right]+E_{P}\left[u_{i}(\bar{x}) \mid A_{n}\right]\right) \\
& =u_{i}\left(x^{*}(0)\right)+\beta_{i} E_{P}\left[u_{i}\left(x^{*}(1)\right)\right]+\beta_{i}\left(E_{P}\left[u_{i}(\bar{x}) \mid A_{n}\right]-E_{P}\left[u_{i}\left(x^{*}(1)\right) \mid A_{n}\right]\right) \\
& =U_{i}\left(x^{*}\right)+\beta_{i}\left(E_{P}\left[u_{i}(\bar{x}) \mid A_{n}\right]-E_{P}\left[u_{i}\left(x^{*}(1)\right) \mid A_{n}\right]\right)
\end{aligned}
$$

By the strict concavity of $u_{i}(\cdot), E_{P}\left[u_{i}(\bar{x}) \mid A_{n}\right]-E_{P}\left[u_{i}\left(x^{*}(1)\right) \mid A_{n}\right]>0$ so that $U_{i}\left(x^{\prime}\right)>U_{i}\left(x^{*}\right)$. But the value of $x^{\prime}$ is given by:

$$
\begin{aligned}
x^{\prime}(0)+E_{Q}\left[\frac{x^{\prime}(1)}{1+r}\right] & =x^{*}(0)+E_{P}\left[\xi \frac{\mathbf{1}_{\Omega \backslash A_{n}} x^{*}(1)+\mathbf{1}_{A_{n}} E_{P}\left[x^{*}(1) \mathbf{1}_{A_{n}}\right]}{1+r}\right] \\
& =x^{*}(0)+E_{P}\left[\xi \frac{x^{*}(1)}{1+r}\right]+E_{P}\left[\xi \frac{\mathbf{1}_{A_{n}} E_{P}\left[x^{*}(1) \mathbf{1}_{A_{n}}\right]}{1+r}\right]-E_{P}\left[\xi \frac{x^{*}(1) \mathbf{1}_{A_{n}}}{1+r}\right]
\end{aligned}
$$

But as $\xi=\xi\left(N^{e}(1)\right)$, there is a constant $\xi_{n}$ such that $\xi$ ProofofTheorem $3.2 A_{n}=$ $\xi_{n}$ ProofofTheorem $3.2 A_{n}$, which implies

$E_{P}\left[\xi \frac{\mathbf{1}_{A_{n}} E_{P}\left[x^{*}(1) \mathbf{1}_{A_{n}}\right]}{1+r}\right]-E_{P}\left[\xi \frac{x^{*}(1) \mathbf{1}_{A_{n}}}{1+r}\right]=\xi_{n} \frac{E_{P}\left[x^{*}(1) \mathbf{1}_{A_{n}}\right]}{1+r}-\xi_{n} \frac{E_{P}\left[x^{*}(1) \mathbf{1}_{A_{n}}\right]}{1+r}=0$

So $x^{\prime}$ is feasible and preferred to $x^{*}$ which contradicts $x^{*}$ being optimal.

\subsection{Proof of Theorem 4.1}

Let us compare the economy without the signal, $\mathcal{E}(\Omega, \mathcal{F}, P)$, with the one with the signal, $\mathcal{E}^{\prime}\left(\Omega^{\prime}, \mathcal{F} \vee \mathcal{F}_{Y}, P^{\prime}\right)$. For any Walrasian equilibrium pair $(\pi, x)$ of $\mathcal{E}$, there is an equivalent Walrasian equilibrium for $\mathcal{E}^{\prime}$, $\left(\pi^{\prime}, x\right)$ where for any event $A \in \mathcal{F}, \pi\left(\mathbf{1}_{A}\right)=\pi^{\prime}\left(\mathbf{1}_{A}\right)$. This follows from the assumption of no initial signal: the addition of the signal is like adding a sunspot to the $\{0,1\}$ state-contingent commodity economy.

If we now add security markets, then the signal is no longer a sunspot, because of the individual non-neutrality assumption. The signal can have two effects: allow for arbitrage opportunities and allow for additional arbitrage-free equilibria.

Nevertheless, if we take the change of measure $\mathrm{d} Q / \mathrm{d} P$ and the interest rate $r$ from Theorem 3.1. The price processes

$$
S_{i}(t)=\frac{E_{Q}\left[L \mid \mathcal{F}_{t}, Y_{t}\right]}{1+r}
$$


are trivially $Q$-martingales on the extended probability space, they do not allow for arbitrage opportunities and by completeness, imply the same measure on $\mathcal{F}$. Hence, they allow the same consumption allocations as those that were available in Theorem 3.1. The only possibility left is that they also allow for better consumption allocations than in Theorem 3.1. But as at date zero, the signal is a mean-preserving spread and $\mathrm{d} Q / \mathrm{d} P$ is a function of the aggregate endowment, the extended budget set adds, at best, mean-preserving spreads of $x$, which (by the concavity of $u_{i}$ ) can never be better than the original $x$. By the aggregate-neutrality assumption, the same aggregate trades are feasible, and as the bond and the insurance trades are also trivially feasible, we are done.

\subsection{Proof of Theorem 4.2}

Consider the economy without the signals. Then, one can apply Theorem 3.1 and obtain a redundant asset equilibrium with full insurance, $k$ fully diversified portfolios and a bond. Let $\left(x_{i}\right)$ denote agent $i$ 's (optimal) allocation in equilibrium and consider the same economy with signals.

Because there is no initial signal and aggregate neutrality the properties of $\tilde{N}$ and the prices of the fully diversified portfolios remain unchanged. Hence, the budget set $B\left(e_{i}, S_{j}\right)$ is the same as the one without signals only smaller. If the $x_{i}$ was the best among those in the budget set, it will continue to be the best if we shrink the budget set, as long as it is in that smaller budget set. But, the new restrictions on feasible trades are that agents cannot dynamically trade insurance. By Theorem 3.1 for all $t \in[0,1]$ it is optimal for $\theta_{i, i}(t)=1$. Hence, the old trading strategy continues to be feasible, which implies that $x_{i} \in B\left(e_{i}, S_{j}\right)$. As this is true for all $i$ we are done. 